\title{
First-in-human trial of multikinase VEGF inhibitor regorafenib and anti-EGFR antibody cetuximab in advanced cancer patients
}

Vivek Subbiah, ${ }^{1}$ Muhammad Rizwan Khawaja, ${ }^{1}$ David S. Hong, ${ }^{1}$ Behrang Amini, ${ }^{2}$ Jiang Yungfang, Hui Liu, ${ }^{1}$ Adrienne Johnson, ${ }^{3}$ Alexa B. Schrock, ${ }^{3}$ Siraj M. Ali, ${ }^{3}$ James X. Sun, ${ }^{3}$ David Fabrizio, ${ }^{3}$ Sarina Piha-Paul, ${ }^{1}$ Siqing Fu, ${ }^{1}$ Apostolia M. Tsimberidou, ${ }^{1}$ Aung Naing, ${ }^{1}$ Filip Janku, ${ }^{1}$ Daniel D. Karp, ${ }^{1}$ Michael Overman, ${ }^{4}$ Cathy Eng, ${ }^{4}$ Scott Kopetz, ${ }^{4}$ Funda Meric-Bernstam, ${ }^{4}$ and Gerald S. Falchook ${ }^{5}$

'Department of Investigational Cancer Therapeutics, Division of Cancer Medicine, ${ }^{2}$ Division of Radiology, The University of Texas MD Anderson Cancer Center, Houston, Texas, USA. ${ }^{3}$ Foundation Medicine, Cambridge, Massachusetts, USA. ${ }^{4}$ Department of GI Medical Oncology, The University of Texas MD Anderson Cancer Center, Houston, Texas, USA. ${ }^{5}$ Drug Development Program, Sarah Cannon Research Institute at HealthONE, Presbyterian/St. Luke's Medical Center, Denver, Colorado, USA.

Role of funding source: The funding organizations had no role in study design, data collection and analysis, decision to publish, or preparation of the manuscript.

Conflict of interest: V. Subbiah received research funding from Novartis, Bayer, GSK, Nanocarrier, Vegenics, Northwest Biotherapeutics, Berghealth, Incyte, Fujifilm, Pharmamar, D3, Pfizer, Amgen, and Abbvie. D.S. Hong received research funding from Novartis, Genentech, Eisai, Astra-Zenica, Pfizer, and MiRNA. $F$. Janku received research funding from Novartis. S. Piha-Paul received research funding from Merck, Puma, Samumed, Curis, Cerulean, Incyte, AbbVie, FivePrime, GlaxoSmithKline, Helix, Biomarin, Bayer, and XuanZhu. A. Johnson, S. Ali, J.X. Sun, D. Fabrizio, and A. Schrock are employees of Foundation Medicine and own stock in the company. F. Meric-Bernstam received research funding from Novartis and Genentech. G. Falchook is an employee of the Sarah Cannon Research Institute at HealthONE.

Submitted: August 29, 2016

Accepted: March 7, 2017

Published: April 20, 2017

Reference information:

JCI Insight. 2017;2(8):e90380.

https://doi.org/10.1172/jici.

insight.90380.
BACKGROUND. The combination of multikinase VEGF inhibitor regorafenib and anti-EGFR antibody cetuximab overcomes intrinsic and acquired resistance in both EGFR-sensitive and EGFR-resistant preclinical models of colorectal cancer (CRC).

METHODS. Utilizing a standard $3+3$ design, a phase I study was designed to determine safety, maximum tolerated dose (MTD), and dose-limiting toxicities (DLTs) of the regorafenib plus cetuximab combination among patients with advanced cancer including CRC. Comprehensive genomic profiling was performed on the exceptional responder.

RESULTS. Among the 27 patients enrolled the median age was 54 years. None of 19 patients treated at dose level 1 (cetuximab i.v. $200 \mathrm{mg} / \mathrm{m}^{2}$ followed by $150 \mathrm{mg} / \mathrm{m}^{2}$ weekly + regorafenib $80 \mathrm{mg}$ daily) experienced a DLT, and 2 of 5 patients treated at dose level 2 (cetuximab i.v. $200 \mathrm{mg} / \mathrm{m}^{2}$ followed by $150 \mathrm{mg} / \mathrm{m}^{2}$ weekly + regorafenib $120 \mathrm{mg}$ daily) experienced a DLT (grade 3 thrombocytopenia $[n=1]$ and grade 3 intra-abdominal bleed $[n=1])$. Most common adverse events were grade 1 or 2 rash (20 patients). Of 24 evaluable patients, 11 (46\%) patients had clinical benefit (stable disease $>6$ cycles or partial response [PR]) (CRC $n=8$, one patient each with head and neck cancer, carcinoma of unknown primary, and glioblastoma). A CRC patient, who progressed on anti-ECFR and regorafenib, achieved a PR (46\% decrease per RECIST v1.1) lasting 15 months. Genomic profiling of an exceptional responder with response for over 27 cycles revealed hypermutated genotype with microsatellite instability (MSI).

CONCLUSION. Regorafenib $80 \mathrm{mg}$ daily plus cetuximab $200 \mathrm{mg} / \mathrm{m}^{2}$ loading dose, followed by 150 $\mathrm{mg} / \mathrm{m}^{2}$ every week is the MTD/recommended phase II dose. The combination demonstrated early signals of activity in wild-type CRC, including 1 exceptional responder with MSI high.

TRIAL REGISTRATION. clinicaltrials.gov NCT02095054

FUNDING. The University of Texas MD Anderson Cancer Center is supported by the NIH Cancer Center Support Grant CA016672. This work was supported in part by the Cancer Prevention Research Institute of Texas grant RP110584 and National Center for Advancing Translational Sciences grant UL1 TR000371 (Center for Clinical and Translational Sciences). 


\section{Table 1. Patient Characteristics on the phase 1 trial with cetuximab and regorafenib}

\begin{tabular}{|c|c|}
\hline & No. of Patients \\
\hline \multicolumn{2}{|l|}{$(n=27)$} \\
\hline \multicolumn{2}{|l|}{ Sex } \\
\hline Male & $16(59 \%)$ \\
\hline Female & $11(41 \%)$ \\
\hline \multicolumn{2}{|l|}{ Age } \\
\hline$\leq 50$ years & 12 (44 \%) \\
\hline$>50$ years & $15(56 \%)$ \\
\hline \multicolumn{2}{|l|}{ Previous Therapies } \\
\hline$<3$ Lines & $7(26 \%)$ \\
\hline$\geq 3$ Lines & $20(74 \%)$ \\
\hline Anti-VEGF Therapy & $22(81 \%)$ \\
\hline Anti-EGFR Therapy & $21(78 \%)$ \\
\hline Phase I Trial Therapy & $5(19 \%)$ \\
\hline Surgery & $17(63 \%)$ \\
\hline Radiation & $10(37 \%)$ \\
\hline \multicolumn{2}{|l|}{ ECOG Performance status } \\
\hline 0 & 3 \\
\hline 1 & 19 \\
\hline 2 & 5 \\
\hline \multicolumn{2}{|l|}{ Diagnoses } \\
\hline Colorectal & $17(63 \%)$ \\
\hline Unknown Primary & $3(11 \%)$ \\
\hline Hepatocellular & $2(7 \%)$ \\
\hline Cervical & $2(7 \%)$ \\
\hline Glioblastoma & $1(3.7 \%)$ \\
\hline Renal Cell & $1(3.7 \%)$ \\
\hline Head and Neck & $1(3.7 \%)$ \\
\hline
\end{tabular}

\section{Introduction}

Angiogenesis and EGFR signaling have now well-established roles in cancer biology. VEGF plays a pivotal role in tumor angiogenesis, while activation of the EGFR has been linked to many processes crucial to tumor progression $(1,2)$. Close relationships exist between these 2 pathways. Preclinical studies suggest that the EGFR may have a role in angiogenesis, and also that inhibition of the EGFR downregulates VEGF (3-6). Converse1y, VEGF upregulation independent of EGFR signaling seems to contribute to resistance to EGFR inhibition (7). Moreover, VEGF inhibition may also block EGFR autocrine signaling and thereby inhibit cancer cell growth (8).

Given that the EGFR and VEGF share common downstream signaling pathways, combined inhibition of these 2 targets may enhance efficacy. In vivo preclinical data have demonstrated decreased angiogenesis as well as increased tumor and endothelial cell apoptosis with combined inhibition of VEGF and EGFR (9).

Inhibitors of VEGF and EGFR have become key therapies in several tumor types. Regorafenib is a multikinase inhibitor, with targets including VEGF receptors 1-3, KIT, and PDGFR- $\alpha$ and $-\beta$. It is approved for use in patients with refractory colon cancer as well was gastrointestinal stromal tumors. Cetuximab is one of the earliest employed monoclonal antibodies targeting the EGFR, and is approved for use in metastatic KRAS wild-type colorectal cancer (CRC) and surgically unresectable squamous cell carcinoma of head and neck. All patients receiving regorafenib or cetuximab eventually progress, and a search for more effective treatments continues.

Recently, Napolitano et al. studied the in vitro effect of the combination of regorafenib plus cetuximab in $K R A S$ - and $B R A F$-mutated human colorectal cell lines as a model of primary resistance to cetuximab as well as colorectal cell line models of acquired resistance to cetuximab (10). The study demonstrated a synergistic antiproliferative and apoptotic effect of this combination by blocking MAPK and AKT pathways, compared with either drug alone or control. The authors then injected nude mice with colorectal cancer cell lines with primary and secondary resistance to cetuximab, and demonstrated significant tumor inhibition with the combination of regorafenib and cetuximab compared with either drug alone or control. Similar results were also observed in an orthotopic $K R A S$-mutated human CRC xenograft.

We hypothesized that the combination of regorafenib and cetuximab may enhance antitumor efficacy. Therefore, this phase I trial was conducted to determine maximum tolerated dose (MTD) and dose-limiting toxicities (DLTs) with this regimen, and to assess the antitumor efficacy of this combination. We also report on comprehensive genomic profiling (CGP) in a patient with CRC and mismatch repair (MMR) deficiency who had an exceptional response of stable disease beyond 20 cycles of therapy. Recently, MMR status was shown to predict response to checkpoint immunotherapy in CRC and non-CRC patients (11). More patients with CRC are being tested for microsatellite instability (MSI) status for checkpoint therapy and treatment options are needed for checkpoint progressors.

\section{Results}

All 27 patients enrolled in the trial were evaluable for toxicity and 24 (89\%) (13 male [54\%]) were evaluable for response, as they completed a full cycle with restaging and were included in the analysis. The median age of patients was 54 (range 28-68) years. Sixty-three percent of the patients had CRC (17 patients). Patients had received a median of 4 (range $0-7$ ) lines of systemic therapies. Twenty patients had received local treatments including surgery, radiation, chemo-embolization and/or radiofrequency ablation. Twenty-two patients had an Eastern Cooperative Oncology Group (ECOG) performance status of 0 or 1. Patient characteristics are summarized in Table 1.

Toxicities. None of the 19 patients treated at dose level 1 experienced any DLTs. Two of 5 patients treated at dose level 2 experienced DLT. Therefore, MTD was considered to have been exceeded at dose level 2, and dose level 1 was determined to be the recommended phase II dose. DLTs included grade 3 thrombocytopenia in 1 patient with a diagnosis of glioblastoma, and grade 3 intra-abdominal bleed in 1 patient with a diagnosis of CRC. Other treatment-related adverse events are summarized in Table 2. The most common toxicities included rash (20 patients), fatigue ( 7 patients), hand-foot syndrome (4 patients), myalgia (4 patients), and nausea (4 patients); all these common toxicities were grade 1 or 2 . 
Table 2. Treatment-related adverse events in the phase 1 trial with cetuximab and regorafenib.

\begin{tabular}{|c|c|c|c|c|c|}
\hline \multirow[t]{2}{*}{ Adverse Event } & \multicolumn{4}{|c|}{ No. of Patients } & \multirow[t]{2}{*}{ Total } \\
\hline & Grade 1 & Grade 2 & Grade 3 & Grade 4 & \\
\hline Anemia & 1 & 1 & & & 2 \\
\hline Anorexia & & 2 & & & 2 \\
\hline Back Pain & & 1 & & & 1 \\
\hline Blurred Vision & 1 & & & & 1 \\
\hline Diarrhea & 2 & & & & 2 \\
\hline EF decrease & & 1 & & & 1 \\
\hline Elevated Amylase & & & 1 & & 1 \\
\hline Elevated Lipase & & & & 1 & 1 \\
\hline Elevated AST ${ }^{\mathrm{A}}$ & 1 & & & & 1 \\
\hline Fatigue & 6 & 1 & & & 7 \\
\hline Intra-Abdominal Bleed & & & 1 & & 1 \\
\hline Leukopenia & & 1 & & & 1 \\
\hline Mucositis & 1 & & & & 1 \\
\hline Myalgia & 3 & 1 & & & 4 \\
\hline Nausea & 4 & & & & 4 \\
\hline Neutropenia & 1 & 1 & & & 2 \\
\hline Proteinuria & 1 & & & & 1 \\
\hline Rash & 17 & 3 & & & 20 \\
\hline Thrombocytopenia & 1 & & 1 & & 2 \\
\hline Vomiting & 2 & & & & 2 \\
\hline Weight Loss & 1 & & & & 1 \\
\hline
\end{tabular}

Patients were able to continue treatment for a median of 5 (range 1-22) cycles; 1 patient is continuing treatment after 27 cycles without any evidence of progression. Thirteen patients received treatment for fewer than 6 cycles, 9 received treatment for 6 to 10 cycles, and 2 for 20 or more cycles.

Responses. Twenty-four patients were evaluable for response. One patient achieved partial response, and 16 had stable disease as best response. Eleven (46\%) of patients had clinical benefit from treatment and included 8 patients with CRC and 1 patient each with squamous cell cancer of head and neck, cancer of unknown primary, and glioblastoma (with EGFR G598V mutation).

Among 17 patients with CRC, all had previously received anti-VEGF as well as anti-EGFR therapy, and 5 had received regorafenib (Table 3). Among these, 7 patients received treatment for fewer than 6 cycles, 6 received treatment for 6 to 10 cycles, and 2 patients for 20 or more cycles. Best response among 14 responseevaluable patients included partial response in 1 patient and stable disease in 10 patients. Eight (53\%) patients with CRC had clinical benefit from treatment. Six had up to a $50 \%$ decrease and 5 had more than a $50 \%$ decrease in serum carcinoembryonic antigen (CEA). Three patients had an increase in CEA. Although we selected for KRAS wild-type CRC, there were no NRAS or atypical KRAS mutations included.

We identified 2 patients who had an exceptional benefit from this treatment for further study. The first patient had CRC and had previously progressed on cetuximab, bevacizumab, and regorafenib as individual monotherapies. The patient achieved a partial radiographic response with a $46 \%$ decrease in tumor measurements per RECIST v1.1 that lasted for approximately 15 months. A 50-gene next-generation sequencing analysis of the tumor specimen revealed a mutation in TP53.

The second patient had CRC with Lynch syndrome (MSI high) and had also progressed on cetuximab, bevacizumab, and regorafenib. The 50-gene analysis of the tumor specimen revealed mutations in the CTNNB1 and FGFR1 genes. Subsequent comprehensive genomic profiling identified alterations 
Table 3. Mutational analysis of patients enrolled in the trial

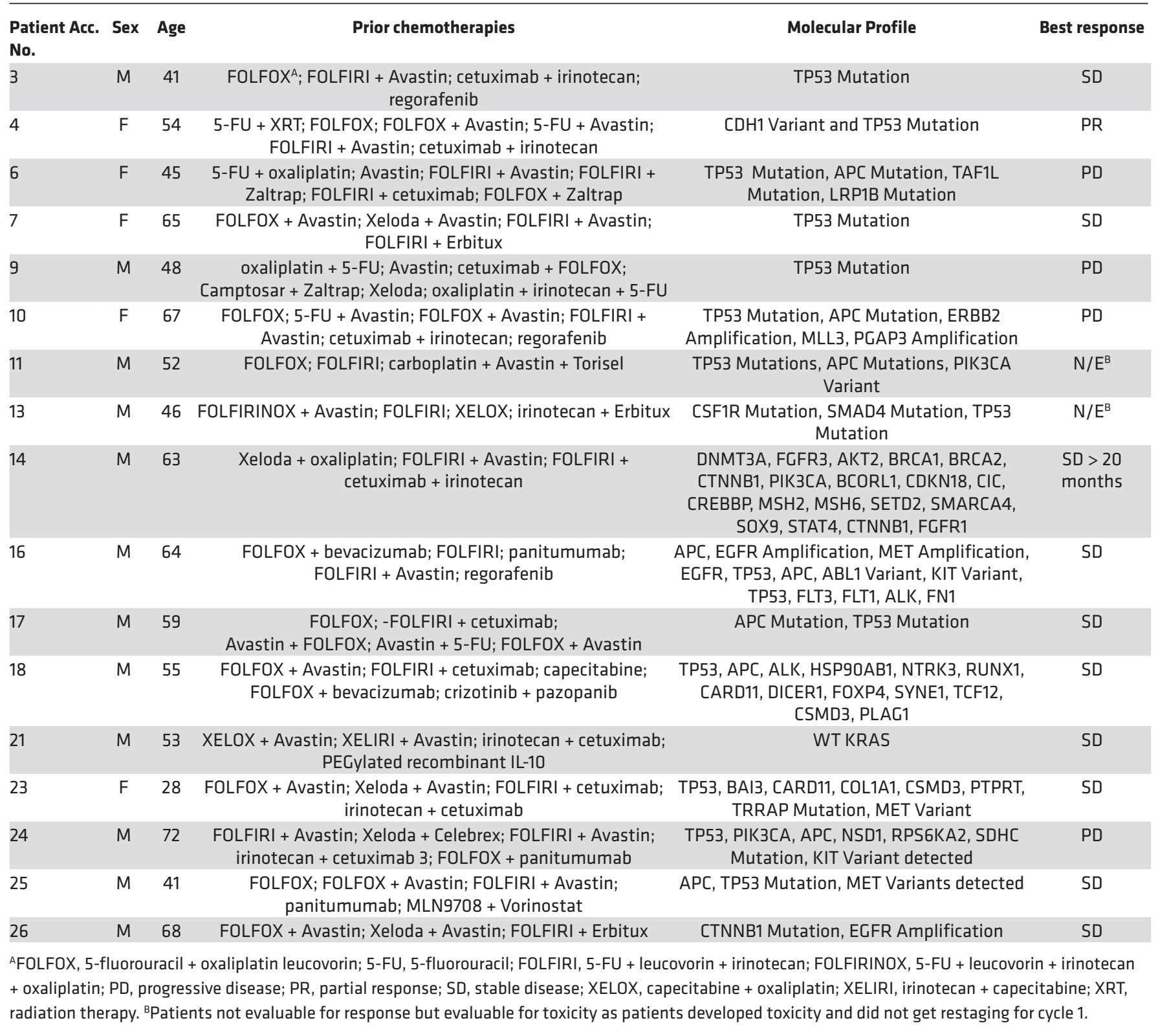

in the AKT2, BRCA1, BRCA2, CTNNB1, DNMT3A, FGFR3, MSH2, MSH6, PIK3CA, CDKN1B, BCORL, CIC, CREBBP, SETD2, SMARCA4, SOX9, and STAT4 genes. These alterations were frameshift mutations and are summarized in Tables 4-6. The mutational burden in this tumor was 99 mutations/ megabase, which exceeds $99.3 \%$ of other tumors (Frampton et al., manuscript in preparation, personal communication). This patient harbors a MSH2 R389* nonsense mutation, and there is an additional MSH2 splice site mutation and an MSH6 frameshift mutation (Tables 4-6). This patient has ongoing stable disease after 20 cycles of treatment (Figure 1).

\section{Discussion}

This open-label phase I trial studied the safety and tolerability of the regorafenib plus cetuximab combination among patients with advanced cancer refractory to several lines of therapy. Dose level 1 was determined to be the MTD, and no patients experienced any DLT at this dose level. Toxicities observed were consistent with previously published literature about these drugs (12-14). 


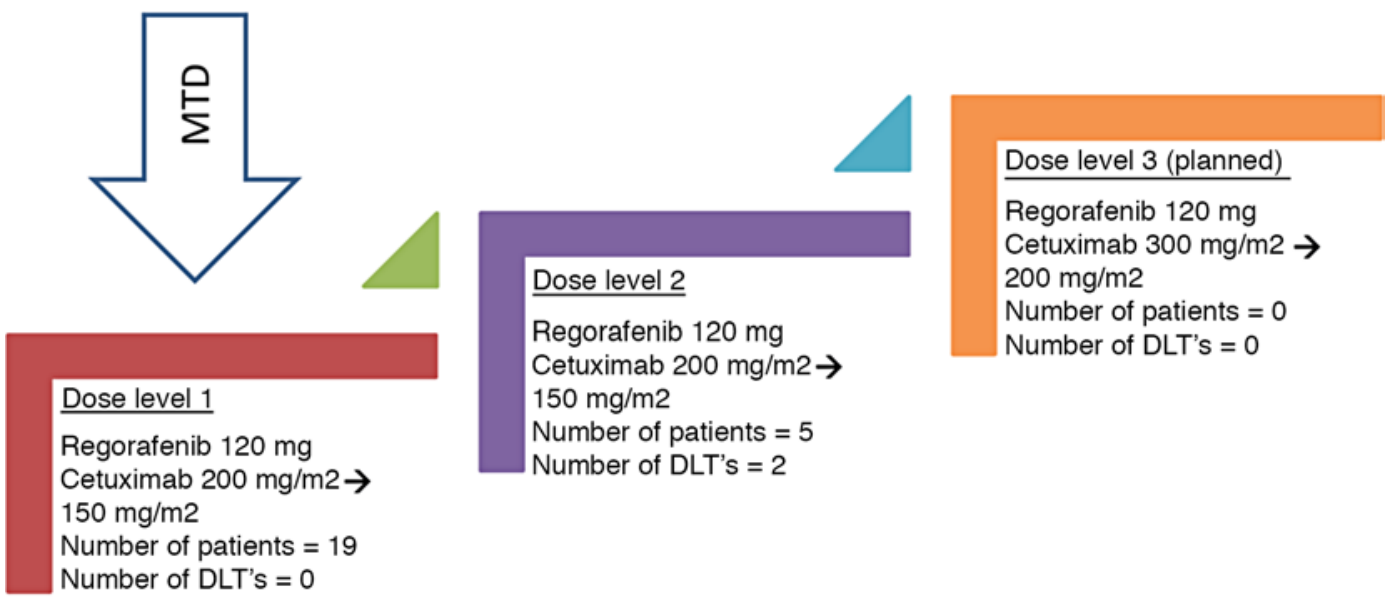

Figure 1. Dose escalation schema showing the number of dose levels, doses, number of patients enrolled, and dose-limiting toxicities (DLTs).

To our knowledge, this is the first trial to study the combination of regorafenib and cetuximab. Previous trials have studied combinations of other anti-VEGF and anti-EGFR agents. Combination of bevacizumab, an anti-VEGF monoclonal antibody, in combination with erlotinib, a small-molecule EGFR inhibitor, in patients with non-small cell lung cancer demonstrated encouraging efficacy and safety profile $(15,16)$.

Although based on a phase II BOND-2 trial, there was initial enthusiasm for combining anti-VEGF plus anti-EGFR therapy with chemotherapy among patients with CRC, which showed encouraging efficacy of a combination of cetuximab, bevacizumab, and irinotecan among bevacizumab-naive irinotecan-refractory patients (17). However, subsequent trials demonstrated less favorable results. The phase II BOND-2.5 trial studied the same combination among bevacizumab-refractory patients and reported modest efficacy (18). Similarly, the CAIRO-2 trial evaluated the addition of cetuximab to chemotherapy plus bevacizumab and

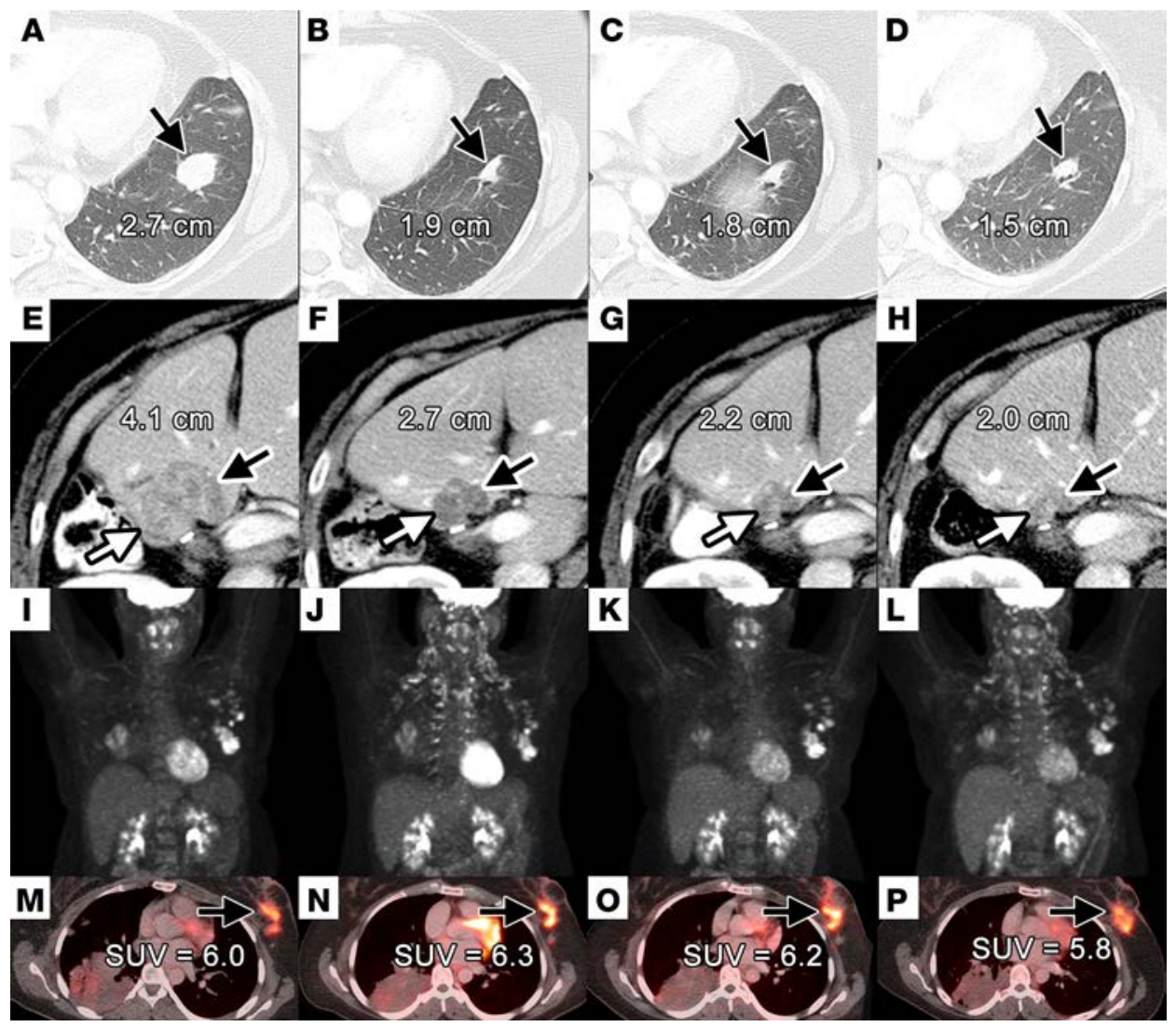

Figure 2. Representative examples of imaging studies of patients with response in the phase 1 trial. Top 2 rows: A 54-year-old woman with metastatic colorectal cancer and partial response. (AD) A left lower lobe nodule decreases in size over the course of the first 3 followup scans, obtained approximately every 6 weeks. (E-H) A right hepatic lobe lesion decreases in size and enhancement over the same time period. Bottom 2 rows: A 35-year-old woman with metastatic carcinoma of unknown primary and stable disease. (I-L) Maximum intensity projections from ${ }^{18} \mathrm{~F}$-FDG PET/CTs obtained approximately every 6 weeks show a left breast mass, left axillary adenopathy, and a right lower lobe metastasis. (M-P) Axial fused images at the level of the left breast mass show stable maximum standard uptake value (SUVmax) when accounting for scan-to-scan variation. The postobstructive consolidation in the right lower lobe related to a right perihilar lesion can also be seen. 
Table 4. Comprehensive genomic profile of patient that had prolonged stable disease and clinical benefit on protocol

\begin{tabular}{|c|c|c|c|}
\hline Gene & CDS $^{A}$ Effect & Protein Effect & Allele Frequency (\%) \\
\hline AKT2 & $1112 G>A$ & $\mathrm{R} 371 \mathrm{H}$ & 10 \\
\hline BRCA1 & 1961_1961delA & $\mathrm{K} 654 \mathrm{fs} * 47$ & 9 \\
\hline CTNNB1 & $110 C>A$ & S37Y & 13 \\
\hline DNMT3A & $2645 G>A$ & $\mathrm{R} 882 \mathrm{H}$ & 12 \\
\hline DNMT3A & $2222 C>T$ & A741V & 9 \\
\hline EPHB6 & $2155 C>T$ & R719W & 9 \\
\hline FANCM & 4414G >C & E1472Q & 5 \\
\hline MSH2 & $1165 C>T$ & $\mathrm{R} 389^{*}$ & 52 \\
\hline PIK3CA & $112 C>T$ & R38C & 2 \\
\hline BCORL1 & 5042_5042delC & P1681fs*20 & 21 \\
\hline BRCA2 & $7480 C>T$ & R2494* & 10 \\
\hline CDKN1B & 192_193insT & Q65fs*60 & 12 \\
\hline CIC & 3138_3138delG & S1048fs* 45 & 10 \\
\hline CREBBP & 3244_3244delA & $11084 f s^{*} 15$ & 12 \\
\hline FGFR3 & $1172 C>T$ & A391V & 8 \\
\hline MSH2 & $792+1 G>A$ & splice site $792+1 G>A$ & 12 \\
\hline MSH6 & 226_226delC & $\mathrm{L} 76 f \mathrm{f}^{*} 5$ & 7 \\
\hline SETD2 & 6181_6182delAG & $\mathrm{D} 2064 \mathrm{fs} * 8$ & 10 \\
\hline SMARCA4 & $3557 \mathrm{C}>\mathrm{T}$ & A1186V & 14 \\
\hline soxg & 916_916delG & V306fs*77 & 15 \\
\hline STAT4 & $1094+2 T>C$ & splice site $1094+2 T>C$ & 7 \\
\hline ABL2 & $2881 C>T$ & $\mathrm{R} 961^{*}$ & 7 \\
\hline $\mathrm{ABL} 2$ & $1743 A>C$ & Q581H & 7 \\
\hline AKT1 & $455 A>C$ & Y152C & 6 \\
\hline АКТЗ & $1435 G>A$ & E479K & 7 \\
\hline APC & $5687 \mathrm{C}>\mathrm{T}$ & S1896I & 14 \\
\hline ARID1A & $1280 C>T$ & P427L & 13 \\
\hline ARID1A & $6203 C>T$ & S2068L & 8 \\
\hline ARID1B & 2165_2165delC & $\mathrm{P} 722 \mathrm{fs} \mathrm{s}^{*} 10$ & 15 \\
\hline ARID1B & 3861_3862delGA & Q1287fs*20 & 7 \\
\hline ATM & $6482 G>A$ & $\mathrm{R} 2161 \mathrm{H}$ & 6 \\
\hline BAP1 & $1552 C>T$ & R518W & 13 \\
\hline BRCA2 & $2880 G>T$ & K960N & 16 \\
\hline BRCA2 & 7507G $>A$ & V2503I & 14 \\
\hline BRIP1 & $1340 A>T$ & N447I & 7 \\
\hline CARD11 & 1657_1657delC & $\mathrm{R} 555 \mathrm{fs} * 45$ & 17 \\
\hline CDC73 & $1537 C>T$ & R513W & 12 \\
\hline CDH5 & $1427 C>T$ & A476V & 7 \\
\hline CDK12 & $3377 A>C$ & Q1126R & 6 \\
\hline
\end{tabular}

${ }^{A} \mathrm{CDS}$, coding sequence. reported worse outcomes with dual antibody therapy; no benefit of dual antibody therapy was observed even among patients with wild-type KRAS colorectal cancer (19). The PACCE trial evaluated the addition of panitumumab, an anti-EGFR antibody, to chemotherapy and bevacizumab, and reported increased toxicity and progression, irrespective of KRAS status (20). Another trial of a phase III randomized, placebo-controlled study of cetuximab plus brivanib alaninate versus cetuximab plus placebo in patients with metastatic, chemotherapy-refractory, wildtype K-RAS CRC was also negative despite positive effects on progression-free survival and objective response (21).

More recently, results were reported for a phase I study of cetuximab with erlotinib and bevacizumab in heavily pretreated cancer patients, most of whom had previously received prior cetuximab as well as bevacizum$a b$. The combination was well tolerated and demonstrated clinical benefit in $34 \%$ patients with CRC (22). A retrospective review of patients treated with EGFR therapy who progressed and rechallenged supported the notion of anti-EGFR retreatment in metastatic CRC (23).

The current study demonstrates that the combination of regorafenib (80 $\mathrm{mg}$ daily for 5 days every week for 3 weeks, followed by a week off) and cetuximab (200 mg/ $\mathrm{m}^{2}$ loading dose, followed by $150 \mathrm{mg} / \mathrm{m}^{2}$ every week) is well tolerated. The combination, although at a lower dose level of the drug, showed a clinical benefit of $46 \%$ and $53 \%$ among all patients and CRC patients, respectively. Among patients with CRC, 79\% had a decrease in CEA and 36\% had a more than $50 \%$ reduction in CEA. These are noteworthy results for patients with advanced CRC, given that all the patients had previously received anti-EGFR as well as anti-VEGF therapy, and several patients had previously received regorafenib. While this trial selected for KRAS wild-type CRC, there were no NRAS or atypical KRAS mutations included in the group, as this is the emerging definition of cetuximab-appropriate patients.

We present a few putative mechanisms underlying the efficacy we observed with dual anti-EGFR and anti-VEGF inhibition in CRC patients. First, unlike earlier studies (1720, 22), we selected only KRAS wild-type CRC patients for this study. Second, the combination of regorafenib and cetuximab may overcome the resistance to cetuximab, as

suggested by preclinical data reported by Napolitano et al. (10). Third, regorafenib is a multikinase inhibitor of VEGF receptors $1-3$ that also targets KIT, PDGFR- $\alpha$ and $-\beta$, RET, FGFR 1 and 2 , TIE2, DDR2, TrkA, Eph2A, RAF-1, BRAF, SAPK-2, PTK5, and ABL. It is plausible that inhibition of one of the other targets of regorafenib contribute to overcoming resistance to previous anti-VEGF or anti-EGFR therapy.

Notably, the exceptional responder with Lynch syndrome may represent a distinct mode of benefit from the combination of cetuximab and regorafenib. Genomic profiling demonstrated an exceptionally high mutational burden even in the context of MSI. The benefit here may be linked to either the very high mutational burden or to the specific mutations in this tumor. Although the other patients in this study were microsatellite stable, CGP was not performed to assess tumor mutational burden (TMB) in these patients to further support this hypothesis, including a lack of characterization of the partial responder patient as to whether this patient had high TMB despite displaying MSI. 
Table 5. Comprehensive genomic profile of patient that had prolonged stable disease and clinical benefit on protocol

\begin{tabular}{|c|c|c|c|}
\hline Gene & CDS $^{A}$ Effect & Protein Effect & Allele Frequency (\%) \\
\hline CIC & 499G>A & G167R & 13 \\
\hline CTCF & $968 \mathrm{C}>\mathrm{A}$ & R323Q & 12 \\
\hline CYLD & $1190 G>A$ & R397H & 13 \\
\hline DNMT3A & $506 G>A$ & R169Q & 6 \\
\hline DNMT3A & $446 C>T$ & A149V & 12 \\
\hline EGFR & 1975G >A & G659R & 8 \\
\hline EPHB1 & 733_734insG & $E 245 f s^{*} 16$ & 7 \\
\hline EPHB4 & 2597G>A & $\mathrm{R} 866 \mathrm{H}$ & 15 \\
\hline EPHB4 & $1120 G>A$ & G374R & 11 \\
\hline EPHB6 & $308 C>T$ & A103V & 12 \\
\hline EPHB6 & $2393 C>T$ & A798V & 7 \\
\hline $\mathrm{EZH} 2$ & $1516 G>A$ & A506T & 6 \\
\hline FANCA & 1304G >A & $\mathrm{R} 435 \mathrm{H}$ & 13 \\
\hline FANCM & $3295 C>T$ & R1099C & 7 \\
\hline FAT3 & $1172 C>A$ & P391H & 13 \\
\hline FAT3 & $1951 C>A$ & L651I & 7 \\
\hline FLT3 & $1014 \mathrm{~A}>\mathrm{T}$ & Q338H & 6 \\
\hline FLT4 & $815 A>G$ & Q272R & 5 \\
\hline H3F3A & $404 G>A$ & $\mathrm{R} 135 \mathrm{H}$ & 10 \\
\hline IGF2R & $2174 C>T$ & T725M & 11 \\
\hline IKZF1 & $551 \mathrm{C}>\mathrm{A}$ & R184Q & 10 \\
\hline INHBA & $514 A>G$ & T172A & 14 \\
\hline INHBA & 95_95delC & $D 34 \mathrm{fs}^{*} 30$ & 8 \\
\hline INSR & $236 C>A$ & $\mathrm{P79H}$ & 7 \\
\hline IRS2 & $2800 C>T$ & P934S & 18 \\
\hline IRS2 & $1853 C>T$ & A618V & 11 \\
\hline JAK1 & $2432 G>A$ & R811K & 12 \\
\hline JAK3 & $94 G>A$ & $\mathrm{~A} 32 \mathrm{~T}$ & 13 \\
\hline KDM5A & $2860 C>T$ & $\mathrm{R} 954^{*}$ & 12 \\
\hline LRP1B & $5845 A>G$ & T1949A & 7 \\
\hline LRP6 & $86 G>A$ & R29Q & 13 \\
\hline MERTK & $764 C>T$ & T255M & 11 \\
\hline MKNK2 & $1033 A>C$ & I345V & 13 \\
\hline MLL & $5225 G>A$ & G1742E & 9 \\
\hline MLL2 & $15491 A>C$ & Q5164R & 7 \\
\hline MLL2 & $4322 G>A$ & $\mathrm{R} 1441 \mathrm{H}$ & 10 \\
\hline MLL2 & $878 C>T$ & T293M & 12 \\
\hline MLL3 & $3865 C>T$ & Q1289* & 11 \\
\hline MYCL1 & 1119_1120insA & R375fs*22+ & 13 \\
\hline MYCL1 & 446G >C & R149P & 14 \\
\hline
\end{tabular}

In this vein, an important followup study would be to apply this treatment regimen to additional MSI-H, high mutational burden CRC patients to assess replication of the response. The clinical need is clear, as although MSI-H CRC patients are known to have a good response rate to programmed cell death protein 1 (PD-1) inhibitors, optimal treatment for such patients is unclear after progression on immunotherapy. The combined cetuximab/regorafenib regimen could also benefit those CRC patients who are de novo refractory to PD-1 treatment or contraindicated from receiving such treatment due to comorbidities such as autoimmune disease. In conclusion, the combination of regorafenib ( $80 \mathrm{mg}$ daily for 3 weeks, followed by a week off) and cetuximab (200 mg/m² loading dose, followed by $150 \mathrm{mg} / \mathrm{m}^{2}$ every week) is reasonably well tolerated and demonstrated early signals of activity. We recommend a larger phase II study with this combination among patients with $K R A S$ wild-type colorectal cancer who have previously received anti-EGFR and anti-VEGF therapy, and an explicit effort to include MSI and/or high TMB patients to replicate the exceptional responder outcome seen here.

\section{Methods}

Patients were eligible if they were aged 12 years or older, had evaluable or measurable advanced or metastatic cancer refractory to standard therapies, and had significant organ function reserve, defined as leukocyte count (WBC) greater than or equal to $3,000 / \mathrm{ml}$, absolute neutrophil count (ANC) greater than or equal to $1,000 / \mathrm{ml}$, platelets greater than or equal to $100,000 / \mathrm{ml}$, creatinine less than or equal to 2 times the upper limit of normal (ULN), total bilirubin less than or equal to 2 times ULN, alanine aminotransferase (ALT, formerly serum glutamic pyruvic transaminase [SGPT]) less than or equal to 3 times ULN, and the ECOG performance status less than or equal to 2. For patients with liver metastases, total bilirubin less than or equal to 3 times the ULN, ALT or aspartate aminotransferase (AST, formerly serum glutamic oxaloacetic transaminase [SGOT]) less than or equal to 5 times the ULN were allowed. Patients with KRAS-mutated CRC, recent history of major surgery, hemoptysis or uncontrolled bleed, uncontrolled hypertension, clinically significant cardiovascular disease, or untreated or uncontrolled brain metastases were excluded.

Using the standard $3+3$ trial design, patients received an escalating dose of regorafenib and cetuximab (Table 1) in 28-day cycles (Figure 2 and Table 7). During each cycle, regorafenib was

administered orally daily for 21 days; patients did not receive any regorafenib during week 4 of each cycle. Cetuximab was administered i.v. every week following an initial loading dose on day 1 of cycle 1.

Three patients were enrolled at the same dose level; if none of them experienced DLT, the next cohort of 3 patients was treated at the next higher dose level. If 1 of 3 patients treated at a dose level experienced DLT, 3 more patients were enrolled at the same dose level. If no other patient experienced DLT, the next cohort of 3 patients was enrolled at the next higher dose level. If 2 or more patients treated at a dose level experienced DLT, the MTD was considered to have been exceeded. In that case, 3 more patients (for a total of 6) were enrolled at the next lower dose level unless 6 patients had already been treated at that dose. In summary, the MTD was defined as the highest dose studied in which the incidence of DLT was less than $33 \%$. 
Table 6. Comprehensive genomic profile of patient that had prolonged stable disease and clinical benefit on protocol

\begin{tabular}{|c|c|c|c|}
\hline Gene & CDS $^{\mathrm{A}}$ Effect & Protein Effect & Allele Frequency (\%) \\
\hline NCOR1 & $5216 G>A$ & R1739Q & 10 \\
\hline NOTCH3 & $4130 c>G$ & A1377G & 53 \\
\hline NSD1 & $5843 G>A$ & $\mathrm{R} 1948 \mathrm{H}$ & 13 \\
\hline NSD1 & 7354G >A & $\mathrm{A} 2452 \mathrm{~T}$ & 13 \\
\hline PRKDC & $9168 \mathrm{G}>\mathrm{T}$ & E3056D & 13 \\
\hline PRKDC & $5126 A>G$ & E1709G & 5 \\
\hline PRSS8 & 157G >A & A53T & 6 \\
\hline PTCH2 & $2524 \mathrm{~A}>\mathrm{G}$ & R842G & 49 \\
\hline RAD54L & $1375 C>T$ & H459Y & 13 \\
\hline soxg & 184_186delAAG & K62del & 6 \\
\hline SPEN & $425 G>A$ & $\mathrm{R} 142 \mathrm{H}$ & 7 \\
\hline SPTA1 & $4520 G>A$ & R1507Q & 13 \\
\hline SPTA1 & $2054 A>G$ & E685G & 5 \\
\hline TAF1 & $4300 G>A$ & V1434M & 24 \\
\hline TВX3 & $2221 G>A$ & A741T & 19 \\
\hline TEK & $3227 \mathrm{C}>\mathrm{A}$ & R1076Q & 15 \\
\hline TNFAIP3 & $2282 G>A$ & $\mathrm{R} 761 \mathrm{H}$ & 5 \\
\hline TNFRSF14 & $58 G>A$ & V20I & 48 \\
\hline TNKS & $952 G>C$ & A318P & 47 \\
\hline TNKS2 & $1235 A>G$ & H412R & 6 \\
\hline XRCC3 & $476 C>T$ & P159L & 17 \\
\hline ZNF217 & $1201 C>T$ & R401W & 15 \\
\hline ZNRF3 & $1700 A>G$ & Y567C & 5 \\
\hline
\end{tabular}

No patients were enrolled in the next dose level until 3 patients enrolled at the previous dose level had completed at least 1 cycle of therapy. If a DLT was observed in a patient, dose escalation did not proceed until a total of 6 patients in the cohort had been assessed for toxicity after 1 cycle. DLTs were defined as adverse events related to the study agents during the first cycle of treatment and included any grade 3 or 4 nonhematologic toxicity (except nausea and vomiting responsive to appropriate regimens, alopecia, or correctable electrolyte imbalances), any grade 4 hematologic toxicity lasting 2 weeks or longer despite supportive care, or any severe or life-threatening complication or abnormality. All toxicities were graded according to Common Terminology Criteria for Adverse Events, version 4.0 (CTCAE v4.0).

Once the MTD was determined, additional patients were enrolled; if $33 \%$ or more of patients at that dose level experienced DLT, then that dose level was to be considered above the MTD. Patients had radiologic restaging after every 2 cycles and were allowed to continue treatment in the absence of disease progression or significant toxicity. Radiographic response or progression was evaluated on the basis of Response Evaluation Criteria in Solid Tumors, version 1.1 (RECIST v1.1) (24). 
Table 7. Planned dose escalation schema, number of patients enrolled in each cohort, and dose-limiting toxicities (DLTs)

\begin{tabular}{|c|c|c|c|c|}
\hline Dose Level & Regorafenib & Cetuximab & No. of Patients & No. of DLTs \\
\hline 1 & $80 \mathrm{mg}$ & $200 \mathrm{mg} / \mathrm{m}^{2} \rightarrow 150 \mathrm{mg} / \mathrm{m}^{2}$ & 19 & 0 \\
\hline 2 & $120 \mathrm{mg}$ & $200 \mathrm{mg} / \mathrm{m}^{2} \rightarrow 150 \mathrm{mg} / \mathrm{m}^{2}$ & 5 & 2 \\
\hline 3 & $120 \mathrm{mg}$ & $300 \mathrm{mg} / \mathrm{m}^{2} \rightarrow 200 \mathrm{mg} / \mathrm{m}^{2}$ & - & - \\
\hline 4 & $160 \mathrm{mg}$ & $300 \mathrm{mg} / \mathrm{m}^{2} \rightarrow 200 \mathrm{mg} / \mathrm{m}^{2}$ & - & - \\
\hline 5 & $160 \mathrm{mg}$ & $400 \mathrm{mg} / \mathrm{m}^{2} \rightarrow 250 \mathrm{mg} / \mathrm{m}^{2}$ & - & - \\
\hline-1 & 80 mg & $100 \mathrm{mg} / \mathrm{m}^{2} \rightarrow 75 \mathrm{mg} / \mathrm{m}^{2}$ & - & - \\
\hline
\end{tabular}

Molecular profiling. All histology was centrally reviewed and molecularly tested at MD Anderson as part of standard of care as previously published (23). Paraffin-embedded tumor sections that were macrodissected were used for DNA extraction. KRAS exon 2 (codons 12 and 13) mutations, whenever done were expanded to also report KRAS, NRAS, BRAF V600E, and PIK3CA mutations. This was based on the standard of care testing results done in accordance with the Clinical Laboratory Improvement Amendment (CLIA)-certified lab (23).

One patient with durable response had biopsy samples submitted as formalin-fixed, paraffin-embedded blocks for CGP. Using FoundationOne, DNA of 315 cancer-related genes plus select introns from 31 genes involved in rearrangements was sequenced to a median depth of coverage of $481 \times$.

Statistics. The primary objective of this study was to assess safety and tolerability as well as to define the MTD of combination treatment with regorafenib and cetuximab in patients with advanced cancer and CRC. Secondary objectives included a preliminary assessment of antitumor efficacy of the combination (objective response by RECIST) and a preliminary assessment of the exceptional responder using clinical next-generation sequencing. Descriptive statistics were performed to report patient characteristics, adverse events, and responses observed. Clinical benefit was defined as objective response or no evidence of progression for 6 or more cycles (partial response or stable disease $\geq 6$ cycles).

Study approval. This was an investigator-initiated, non-company sponsored, open-label phase I dose escalation trial conducted at The University of Texas MD Anderson Cancer Center. Informed written consent was obtained from all patients, and the study was reviewed and approved by the IRB of MD Anderson Cancer Center.

\section{Author contributions}

VS and GSF were principal investigators of the phase 1 trial; they designed, conducted, and provided financial and administrative support for the trial, wrote the manuscript, critically revised the manuscript, and submitted the revisions. VS, MRK, SMA, and GSF analyzed the data and wrote the manuscript. VS, MRK, DSH, BA, JY,HL, SP, SF, AMT, AN, FJ, DDK, MO, CE, SK, and FMB enrolled patients in the clinical trial and analyzed data. AJ, ABS, SMA, JXS, and DF performed the next-generation sequencing analysis for the exceptional responder and analyzed the data. All authors were involved in critical analysis of the manuscript and approved the final version of the manuscript.

\section{Acknowledgments}

The authors wish to thank the motivated patients who were enrolled in this clinical trial. We wish to acknowledge the support from "The Beck Family" (Robert Beck and Merry Beck research funds) for Clinical Trial Research Funding. The University of Texas MD Anderson Cancer Center is supported by the National Institutes of Health Cancer Center Support Grant CA016672.

Address correspondence to: Vivek Subbiah, The University of Texas MD Anderson Cancer Center, 1515 Holcombe Boulevard, Unit 455, Houston, Texas 77030, USA. Phone: 713.792.6620; E-mail: vsubbiah@mdanderson.org.

1. Yarden Y, Sliwkowski MX. Untangling the ErbB signalling network. Nat Rev Mol Cell Biol. 2001;2(2):127-137.

2. Hicklin DJ, Ellis LM. Role of the vascular endothelial growth factor pathway in tumor growth and angiogenesis. J Clin Oncol. 2005;23(5):1011-1027.

3. Kim SJ, Uehara H, Karashima T, Shepherd DL, Killion JJ, Fidler IJ. Blockade of epidermal growth factor receptor signaling in 
tumor cells and tumor-associated endothelial cells for therapy of androgen-independent human prostate cancer growing in the bone of nude mice. Clin Cancer Res. 2003;9(3):1200-1210.

4. de Jong JS, van Diest PJ, van der Valk P, Baak JP. Expression of growth factors, growth-inhibiting factors, and their receptors in invasive breast cancer. II: Correlations with proliferation and angiogenesis. J Pathol. 1998;184(1):53-57.

5. Petit AM, et al. Neutralizing antibodies against epidermal growth factor and ErbB-2/neu receptor tyrosine kinases downregulate vascular endothelial growth factor production by tumor cells in vitro and in vivo: angiogenic implications for signal transduction therapy of solid tumors. Am J Pathol. 1997;151(6):1523-1530.

6. Hirata A, et al. ZD1839 (Iressa) induces antiangiogenic effects through inhibition of epidermal growth factor receptor tyrosine kinase. Cancer Res. 2002;62(9):2554-2560.

7. Vallböhmer D, et al. Molecular determinants of cetuximab efficacy. J Clin Oncol. 2005;23(15):3536-3544.

8. Ciardiello F, et al. Antitumor effects of ZD6474, a small molecule vascular endothelial growth factor receptor tyrosine kinase inhibitor, with additional activity against epidermal growth factor receptor tyrosine kinase. Clin Cancer Res. 2003;9(4):1546-1556.

9. Shaheen RM, et al. Inhibited growth of colon cancer carcinomatosis by antibodies to vascular endothelial and epidermal growth factor receptors. Br J Cancer. 2001;85(4):584-589.

10. Napolitano S, et al. Primary and acquired resistance of colorectal cancer to anti-EGFR monoclonal antibody can be overcome by combined treatment of regorafenib with cetuximab. Clin Cancer Res. 2015;21(13):2975-2983.

11. Le DT, et al. PD-1 blockade in tumors with mismatch-repair deficiency. N Engl J Med. 2015;372(26):2509-2520.

12. Grothey A, et al. Regorafenib monotherapy for previously treated metastatic colorectal cancer (CORRECT): an international, multicentre, randomised, placebo-controlled, phase 3 trial. Lancet. 2013;381(9863):303-312.

13. Li J, et al. Regorafenib plus best supportive care versus placebo plus best supportive care in Asian patients with previously treated metastatic colorectal cancer (CONCUR): a randomised, double-blind, placebo-controlled, phase 3 trial. Lancet Oncol. 2015;16(6):619-629

14. Jonker DJ, et al. Cetuximab for the treatment of colorectal cancer. N Engl J Med. 2007;357(20):2040-2048

15. Herbst RS, et al. Phase II study of efficacy and safety of bevacizumab in combination with chemotherapy or erlotinib compared with chemotherapy alone for treatment of recurrent or refractory non small-cell lung cancer. J Clin Oncol. 2007;25(30):4743-4750.

16. Herbst RS, et al. Phase I/II trial evaluating the anti-vascular endothelial growth factor monoclonal antibody bevacizumab in combination with the HER-1/epidermal growth factor receptor tyrosine kinase inhibitor erlotinib for patients with recurrent non-small-cell lung cancer. J Clin Oncol. 2005;23(11):2544-2555.

17. Saltz LB, et al. Randomized phase II trial of cetuximab, bevacizumab, and irinotecan compared with cetuximab and bevacizumab alone in irinotecan-refractory colorectal cancer: the BOND-2 study. J Clin Oncol. 2007;25(29):4557-4561.

18. Segal NH, et al. Phase II study of bevacizumab in combination with cetuximab plus irinotecan in irinotecan-refractory colorectal cancer (CRC) patients who have progressed on a bevacizumab-containing regimen (the BOND 2.5 study). J Clin Oncol. 2009;27(15Suppl):4087-4087.

19. Tol J, et al. Chemotherapy, bevacizumab, and cetuximab in metastatic colorectal cancer. N Engl J Med. 2009;360(6):563-572

20. Hecht JR, et al. A randomized phase IIIB trial of chemotherapy, bevacizumab, and panitumumab compared with chemotherapy and bevacizumab alone for metastatic colorectal cancer. J Clin Oncol. 2009;27(5):672-680.

21. Siu LL, et al. Phase III randomized, placebo-controlled study of cetuximab plus brivanib alaninate versus cetuximab plus placebo in patients with metastatic, chemotherapy-refractory, wild-type K-RAS colorectal carcinoma: the NCIC Clinical Trials Group and AGITG CO.20 Trial. J Clin Oncol. 2013;31(19):2477-2484.

22. Falchook GS, et al. Dual EGFR inhibition in combination with anti-VEGF treatment in colorectal cancer. Oncoscience. 2014;1(8):540-549.

23. Liu X, et al. Retreatment with anti-EGFR based therapies in metastatic colorectal cancer: impact of intervening time interval and prior anti-EGFR response. BMC Cancer. 2015;15:713.

24. Eisenhauer EA, et al. New response evaluation criteria in solid tumours: revised RECIST guideline (version 1.1). Eur J Cancer. $2009 ; 45(2): 228-247$ 